\title{
National trends of childhood vaccination coverage in Bangladesh: Understanding gaps from 2011, 2014 and 2017-18 Demographic and Health Surveys data
}

\section{Satyajit Kundu ( $\square$ satyajitnfs@gmail.com )}

Department of Biochemistry and Food Analysis, Patuakhali Science and Technology University, Patuakhali - 8602, Bangladesh https://orcid.org/0000-0001-9610-1479

\section{Subarna Kundu}

Statistics Discipline, Khulna University, Khulna, Bangladesh

\section{Bright Opoku Ahinkorah}

School of Public Health, Faculty of Health, University of Technology Sydney, Sydney, Australia.

\section{Abdul-Aziz Seidu}

College of Public Health, Medical and Veterinary Services, James Cook University, Australia

\section{Joshua Okyere}

Department of Population and Health, University of Cape Coast, Cape Coast, Ghana.

\section{Susmita Ghosh}

Department of Food Technology and Nutrition Science, Noakhali Science and Technology University, Noakhali, Bangladesh

Ahmed Hossain

Department of Public Health, North South University, Dhaka, Bangladesh.

\section{Najim Z. Alshahrani}

Department of Family and Community Medicine, Faculty of Medicine, University of Jeddah, Jeddah 21589, Saudi Arabia

\section{Md. Hasan Al Banna}

Department of Food Microbiology, Patuakhali Science and Technology University, Patuakhali- 8602, Bangladesh

\section{Research Article}

Keywords: Childhood vaccination, Immunization, Demographic and Health Survey data, Prevalence, Bangladesh

Posted Date: July 16th, 2021 
DOl: https://doi.org/10.21203/rs.3.rs-722674/v1

License: (c) (1) This work is licensed under a Creative Commons Attribution 4.0 International License. Read Full License 


\section{Abstract \\ Background}

Vaccination is a safe and cost-effective strategy for protecting children from life-threatening diseases. This study aimed to investigate the changes over time in proportion of vaccination coverage across demographic subgroups in Bangladesh.

\section{Method}

Vaccination coverage for children 12-59 months of age was obtained from Bangladesh's 2011, 2014, and 2017-18 Demographic and Health Surveys. Three multivariable binary logistic regression models with complete vaccination status at each survey data were used to generate adjusted odds ratios to uncover immunization variations across socio-demographic categories. Further, to detect potential geographic disparities, changes over time were analyzed at both the country and district levels.

\section{Findings}

Vaccination coverage grew from $88.1 \%$ in 2011 to $89.2 \%$ in $2017-2018$, indicating a gap of more than ten percent for complete immunization coverage among children aged 12-59 months. Children aged 2335 were more likely to have full vaccination compared to those aged $12-23$ in 2011 ( $\mathrm{AOR}=1.6,95 \% \mathrm{Cl}=$ 1.3-1.9), $2014(\mathrm{AOR}=1.6,95 \% \mathrm{Cl}=1.3-2.0)$, and 2017-18 (AOR = 1.4, 95\% Cl=1.1-1.7). The likelihood of full vaccination of children increased with maternal education with the highest odds among women with higher education in 2011 ( $\mathrm{AOR}=7.2,95 \% \mathrm{Cl}=4.0-12.9), 2014(\mathrm{AOR}=4.0,95 \% \mathrm{Cl}=2.7-5.9)$, and 2017-18 $(A O R=5.8,95 \% \mathrm{Cl}=3.5-9.6)$ compared to those with no formal education. Children born to mothers who lived in urban areas were more likely to have full vaccination in 2011 ( $A O R=1.5,95 \% \mathrm{Cl}=1.3-1.8), 2014$ $(A O R=1.4,95 \% \mathrm{Cl}=1.2-1.7)$, and $2017-18(A O R=1.4,95 \% \mathrm{Cl}=1.1-1.7)$ compared to those who lived in rural areas. Children born to mothers who had at least 4 ANC visits were more likely to have full vaccination compared to those with less than $3 \mathrm{ANC}$ visits in 2011 ( $\mathrm{AOR}=1.6,95 \% \mathrm{Cl}=1.2-2.0$ ), 2014 $(\mathrm{AOR}=1.5,95 \% \mathrm{Cl}=1.2-2.0)$, and $2017-18(\mathrm{AOR}=1.6,95 \% \mathrm{Cl}=1.2-2.1$ During these three surveys, Rangpur division had the highest vaccine coverage rate, while Sylhet division had the lowest vaccination coverage.

\section{Conclusion}

Although there was an improvement in these nationally representative surveys from 2011 to 2017-18, a portion of children still needs to be vaccinated to ensure full immunization coverage. To achieve $100 \%$ immunization coverage for all Bangladeshi children, policymakers must integrate vaccine programs with personalized health messaging and assurances of health safety for impoverished children and low- 
educated mothers. Increased institutional deliveries and prenatal care visits by mothers could assist increase their children's vaccination coverage.

\section{Introduction}

Promoting the health and wellbeing of children is one of the greatest responsibilities that nations, regions, communities and households have had to bear over the years. Protecting children against diseases constitutes one of the fundamental concerns in the promotion of children's health and wellbeing. This could come in varied ways including the provision of safe drinking water, meeting the nutritional needs of children, as well as vaccinating children against preventable diseases $[1,2]$. This study emphasizes childhood vaccination as a technique for improving children's health and well-being.

Vaccinations are widely acknowledged as one of the safest and most cost-effective ways to protect children against infectious diseases such as tuberculosis and measles [3]. Thus, childhood vaccination has been increasing over the past decades [4]. For example, the vaccination of children against diphtheria-tetanus-pertussis (DTP3) increased astoundingly from a global coverage of $20 \%$ in 1980 to $85 \%$ in 2019 [5]. More profound is the evidence that vaccination averts between 2-3 million deaths attributable to vaccine-preventive diseases such as Diphtheria, Pertussis, Tetanus, and Measles among children under-five every year $[6,7]$.

Although the world has seen remarkable improvements in childhood vaccination, achieving complete coverage over time remains an important public health concern [8]. Not every child is getting vaccinated. For instance, 19.4 million infants did not receive basic vaccination as at the end of 2019 [9,10]. Most of these deficiencies in childhood vaccination coverage are recorded in low-and-middle-income countries (LMICs). Evidence from Ethiopia suggests that in 2018, more than 1.2 million children were not vaccinated with the first dose of measles vaccines [11]. The WHO asserts that, in remote rural areas of LMICs, only 1 out of 20 children have access to vaccination [12].

The consequences of not achieving complete childhood vaccination cannot be underestimated. Vaccination provides an opportunity to avert millions of deaths and a host of vaccine-preventable diseases among children [3]. Within the framework of the WHO, children who miss scheduled vaccinations for any reason due to health facility problems such as canceled vaccination schedules or vaccine stock-outs are categorized as having incomplete vaccination [13]. Denying children access to a complete dose of vaccines would be catastrophic as a countless number of children will die or develop some form of disabilities [14]. As such, it is imperative to understand the nuances that characterize childhood vaccination coverage over time.

Available evidence suggests that there are several factors that influence the uptake of vaccination for children under-five. For instance, a qualitative study by Jalloh et al. [15] indicates that perceived beliefs about the side effects coupled with concerns about receiving multiple vaccines on the same day were significant barriers to the uptake of childhood vaccination and its coverage. Also, other studies from 
Ghana [16] and Nigeria [17] suggest that urban residency and higher maternal education are associated with higher likelihoods of complete childhood vaccination coverage.

Since 1979, the Government of Bangladesh (GOB) has started vaccinations against six preventable diseases (tuberculosis; diphtheria, pertussis, and tetanus; polio; and measles) through the Expanded Program on Immunization (EPI) [18]. According to the Bangladesh Immunization guidelines, children who have received one dose of the vaccine against tuberculosis, Bacille Calmette-Guerin (BCG), three doses of a pentavalent vaccine (DPT, Hib, and $\mathrm{HepB}$ ), three doses of the polio vaccine (excluding the polio vaccine given at birth), and one dose of the measles and rubella vaccine are considered as fully vaccinated, if they would miss any of the recommended doses they will be considered as partially vaccinated $[18,19]$.

Bangladesh as a country has attained significant heights in reducing childhood mortality; this is seen in the country's capacity to meet the Millennium Development Goal 4 [20]. Through the implementation of the WHO's Expanded Programme on Immunization (EPI), Bangladesh was able to commit sufficiently towards the promotion of childhood vaccination coverage which saw a sustained impact on childhood mortality, reducing it from 133 deaths per 1000 live births in 1993 to 46 deaths per 1000 in 2014 [20]. In a bid to augment efforts toward childhood vaccination coverage, the Bangladeshi government came up with three nationwide supplementary immunization activities (SIAs) from 2000 to 2016 [21]. These initiatives resulted in a significant decline in the incidence of measles, from 14745 incident cases in 2010 to 972 in 2016 [15,21]. Nevertheless, Bangladesh faces high levels of childhood mortality which places the country among the top ten countries with the highest rates of childhood mortality, with vaccinepreventable diseases being the causes of these mortalities $[15,22]$. This makes Bangladesh an opportune context to understand childhood vaccine coverage and its concomitant factors.

Bangladesh relies on composite estimates based on administrative coverage data gathered from healthcare providers, population-based household surveys, and governmental agencies [20]. However, due to the incompleteness and mistakes associated with the original collection of data on childhood immunization in Bangladesh, such estimates are frequently incorrect [20]. As a result, utilizing a nationally representative survey provides much more clarity and strong data to investigate the factors that influence vaccination coverage [23]. Using nationally representative data from the BDHS in 2011, 2014 , and 2017-18, the study aims to track the vaccination status of children aged 12 to 59 months and examine the factors that influence full immunization coverage.

\section{Methods}

\section{Study population}

The current study utilized three recent nationally representative cross-sectional Bangladesh demographic health survey data (BDHS) from 2011 to 2017-18. Bangladesh is a south-eastern Asian country that has administratively divided into eight divisions: Dhaka, Chittagong, Khulna, Mymensingh, Rajshahi, Rangpur and Sylhet. The survey included both urban and rural households from all administrative regions. The 
data was collected using two-stage stratified cluster sampling design of the household. At the first stage, enumeration areas were selected with probability proportional to sizes like 672 in 2017-18, 600 in both 2014 and 2011 BDHS respectively. After getting the enumeration area, on average 30 households were selected from each cluster using systematic sample selection. Detailed descriptions of the DHS sampling design were published with the BDHS survey report $[19,24,25]$.

\section{Outcome assessment}

The outcome of interest in this study was to look over the Child vaccination status among children aged 12-59 months in Bangladesh. The government of Bangladesh started the Expanded Program on Immunization (EPI) against six preventable diseases: tuberculosis, especially the extrapulmonary forms (Bacille Calmette-Guérin [BCG] vaccine); diphtheria, pertussis, and tetanus (DPT vaccine); poliomyelitis (oral polio vaccine [OPV]); and measles (measles vaccine) among children in 1979 [24]. Table 1 shows the basic vaccine schedule by time. Children aged greater than 12 months are considered to be fully vaccinated if they have the BCG vaccine, three doses of polio, three doses of DPT and one dose of measles at any time before the survey. Partially vaccinated were defined as lacking any dose of the basic vaccination. While those who failed to take the recommended doses of vaccine were categorized as "none". Vaccination coverage information was collected in two ways from the vaccination card or from the mother's verbal report. For logistic regression, binary vaccination status was recoded as fully vaccinated and not fully vaccinated (merging partially vaccinated and no vaccinated). Hepatitis $B$ vaccine (1-3 dose), Haemophilus influenzae type B vaccine, inactivated polio vaccine (IPV) was not included in the current study.

\section{Explanatory variables}

According to the guidance of reviewed literature and the availability of the variables, several demographic and health variables were included in this current analysis $[3,26,27]$. The included variables for this study are child age, mother's age, mother's education, antenatal care (ANC) visit, place of residence, division, gender, place of delivery, number of children. Children aged 12-59 months were selected to conduct the current study who were categorized as $12-23$ months, 23-35 months, 36-47 months, and 48-59 months. Maternal age was categorized as less than 24 years, 24-34 years, and above 34 years [28,29]. The household wealth index was calculated using principal component analysis of the different household assets, including floor type, lighting source, cooking fuel, main drinking water source, stool disposal facility, stool disposal site, ownership of telephone, internet access, and household head's education, etc [25] The wealth index of the household of the respondent was recategorized as poor (poorest or poorer), middle, and rich (richer, richest). Media access is an important factor for vaccination coverage which was measured by asking mothers about the number of times they read a newspaper, listen to the radio, and watch television. Adding these variables media access was recategorized as less than once a week or at least once a week. For huge non-response missing information for the variable place of 
delivery and antenatal care visits, those respondents were categorized in the other category. Place of delivery was recategorized as home, health facility delivery (public and private health care facility), and others (others, missing). Respondents were categorized into at least 4 visits, less than 4 visits and missing (who does not respond). Maternal education was also categorized into no education, primary education, and secondary or higher. Bangladesh has administratively divided in eight divisions: Dhaka, Chittagong, Khulna, Mymensingh, Rajshahi, Rangpur and Sylhet. Mymensingh division is created in 2015 composing some districts from northern part of Dhaka division [30]. That's why information of Mymensingh division was not available separately in 2011 and 2014 BDHS survey data.

\section{Statistical analysis}

Statistical analysis was conducted considering the children aged 12-59 months in the three recent BDHS data sets 2011, 2014 and 2017-18. To assess the changes in all basic vaccination coverage across Bangladesh, vaccination status among children aged 12-59 months for each vaccine (full, partial and none) among different survey datasets were calculated with chi-square analysis using the three survey datasets to assess the differences. The study used descriptive statistics to show the distribution of the respondents by their characteristics and the differences in the coverage between categories were tested using chi-square analysis. To explore the relationship and changes between children's age and vaccination coverage, the prevalence of fully vaccinated was plotted against their birth year which helps to assess possible cohort effects on vaccination status with a significant test (chi-square test) for trend. The graphical presentation was done in the two populations: first who have a vaccination card and then, for children with a vaccination card or had information from mother's recall. Additionally, to explore the spatial distribution and changes of this distribution over time of vaccination coverage among children aged 12-59 months in the three recent survey year maps of the rate of change of fully vaccinated over time children were created for three survey datasets separately which shows the changes in the rate of fully vaccinated children within each division. The rate of change in the vaccination coverage over time within each division was calculated using the formula:

\section{$\frac{((\% \text { fully vaccinated in the recent year })-(\% \text { fully vaccinated in the previous year }))}{(\% \text { fully vaccinated in the previous year })} \times 100$}

Further, three multivariable binary (fully vaccinated and not fully vaccinated) logistic regression model was used separately to estimate odds ratios with $95 \%$ confidence intervals for socioeconomic factors' association with full vaccination using three different survey datasets. A $5 \%$ significance level was used for each analysis in this study. All analyses were performed using the statistical package SPSS (version 23.0) and STATA (version16.0). Spatial analysis along with maps was generated using ArcGIS (version 10.5).

\section{Ethical approval}


This study used a secondary data analysis of publicly available survey data from the MEASURE DHS program (https://www.dhsprogram.com). Participants in the DHS gave informed consent at the time of participation in the original survey. These DHS survey reports are publicly available; datasets are accessible upon application. We requested the data set, and permission was granted to download and use the data for this study.

\section{Results}

Table 1 shows the basic vaccination administration schedule for children under 12 months in Bangladesh.

Table 1. Basic vaccination administration schedule for children under 12 months in Bangladesh

\begin{tabular}{|l|l|l|}
\hline Vaccine & Disease & Age \\
\hline BCG & Tuberculosis & At birth \\
\hline OPV & Polio & $6,10,14$ weeks \\
\hline DTP & Diphtheria, Tetanus, Pertussis & $6,10,14$ weeks \\
\hline Measles & Measles & 9 months \\
\hline BCG, Bacille Calmette-Guerin; OPV, oral polio vaccine \\
\hline
\end{tabular}

Table 2. Vaccination coverage estimates for Bangladesh demographic and health survey respondents 12-59 months of age by survey year 


\begin{tabular}{|c|c|c|c|c|c|c|c|}
\hline & \multicolumn{2}{|c|}{$2011 ;(N=6678)$} & \multicolumn{2}{|c|}{$2014 ;(N=6105)$} & \multicolumn{2}{|c|}{ 2017-18; $(N=3321)$} & \multirow[t]{2}{*}{ p value } \\
\hline & $\mathrm{n}$ & $\%(95 \% \mathrm{CI})$ & $\mathrm{n}$ & $\%(95 \% \mathrm{CI})$ & $\mathrm{n}$ & $\%(95 \% \mathrm{CI})$ & \\
\hline \multicolumn{8}{|l|}{ Vaccination card $^{\text {a }}$} \\
\hline Yes, seen & 3743 & $56.1(54.9,57.2)$ & 3784 & $62.0(60.8,63.2)$ & 2361 & $71.1(69.5,72.6)$ & \multirow[t]{4}{*}{$<0.001$} \\
\hline Yes, Not seen & 1958 & $29.3(28.2,30.4)$ & 1948 & $31.9(30.7,33.1)$ & 609 & $18.3(17.0,19.7)$ & \\
\hline No longer has card & 688 & $10.3(9.6,11.0)$ & 172 & $2.8(2.4,3.3)$ & 231 & $7.0(6.1,7.9)$ & \\
\hline No card & 288 & $4.3(3.8,4.8)$ & 200 & $3.3(2.9,3.8)$ & 120 & $3.6(3.0,4.3)$ & \\
\hline \multicolumn{8}{|c|}{ Reported vaccinations from vaccination card or mother recall } \\
\hline $\mathrm{BCG}^{\mathrm{a}}$ & 6493 & $97.2(96.8,97.6)$ & 5906 & $96.8(96.3,97.2)$ & 3269 & $98.4(98.0,98.8)$ & $<0.001$ \\
\hline Polio $1^{\mathrm{b}}$ & 6503 & $97.4(97.0,97.8)$ & 5900 & $96.6(96.2,97.1)$ & 3261 & $98.2(97.7,98.6)$ & $<0.001$ \\
\hline Polio $2^{\mathrm{C}}$ & 6392 & $95.8(95.3,96.3)$ & 5814 & $95.3(94.7,95.8)$ & 3217 & $96.9(96.3,97.5)$ & 0.001 \\
\hline Polio $3^{\mathrm{C}}$ & 6237 & $93.5(92.9,94.1)$ & 5649 & $92.6(92.0,93.3)$ & 3142 & $94.7(93.8,95.4)$ & 0.001 \\
\hline \multicolumn{8}{|c|}{ Polio vaccination completion (OPV 1-3) ${ }^{b}$} \\
\hline Full & 6236 & $93.4(92.8,94.0)$ & 5646 & $92.5(91.8,93.1)$ & 3140 & $94.5(93.7,95.3)$ & \multirow[t]{3}{*}{$<0.001$} \\
\hline Partial & 267 & $4.0(3.5,4.5)$ & 255 & $4.2(3.7,4.7)$ & 122 & $3.7(3.0,4.4)$ & \\
\hline None & 174 & $2.6(2.2,3.0)$ & 204 & $3.3(2.9,3.8)$ & 59 & $1.8(1.4,2.3)$ & \\
\hline DTP $1^{\mathrm{b}}$ & 6489 & $97.2(96.8,97.6)$ & 5877 & $96.3(95.8,96.7)$ & 3265 & $98.3(97.8,98.7)$ & $<0.001$ \\
\hline DTP $2^{\mathrm{d}}$ & 6379 & $95.6(95.0,96.0))$ & 5795 & $94.9(94.4,95.5)$ & 3237 & $97.5(96.9,98.0)$ & $<0.001$ \\
\hline DTP $3^{\mathrm{d}}$ & 6218 & $93.2(92.5,93.7)$ & 5620 & $92.1(91.4,92.7)$ & 3183 & $95.8(95.1,96.5)$ & $<0.001$ \\
\hline \multicolumn{8}{|c|}{ DTP vaccination completion (DTP 1-3) ${ }^{b}$} \\
\hline Full & 6217 & $93.1(92.5,93.7)$ & 5618 & $92.0(91.3,92.7)$ & 3183 & $95.8(95.1,96.5)$ & \multirow[t]{3}{*}{$<0.001$} \\
\hline Partial & 272 & $4.1(3.6,4.6)$ & 259 & $4.2(3.8,4.8)$ & 82 & $2.5(2.0,3.1)$ & \\
\hline None & 188 & $2.8(2.4,3.2)$ & 228 & $3.7(3.3,4.2)$ & 56 & $1.7(1.3,2.2)$ & \\
\hline Measles $^{\mathrm{e}}$ & 6006 & $90.0(89.3,90.7)$ & 5321 & $87.3(86.5,88.2)$ & 21.1 & $91.2(90.1,92.1)$ & $<0.001$ \\
\hline \multicolumn{8}{|l|}{ Vaccination status ${ }^{f}$} \\
\hline Full & 5886 & $88.1(87.3,88.9)$ & 5194 & $85.1(84.2,86.0)$ & 2961 & $89.2(88.0,90.2)$ & \multirow[t]{3}{*}{$<0.001$} \\
\hline Partial & 636 & $9.5(8.8,10.3)$ & 737 & $12.1(11.3,13.0)$ & 312 & $9.4(8.4,10.5)$ & \\
\hline None & 156 & $2.3(2.0,2.7)$ & 174 & $2.9(2.5,3.3)$ & 48 & $1.4(1.1,1.9)$ & \\
\hline \multicolumn{8}{|c|}{$\begin{array}{l}\text { a } 2011 \mathrm{n}=6677 ; 2014 \mathrm{n}=6104 ; 2017-18 \mathrm{n}=3321 . \\
\text { b } 2011 \mathrm{n}=6677 ; 2014 \mathrm{n}=6105 ; 2017-18 \mathrm{n}=3321 \text {. } \\
\text { c } 2011 \mathrm{n}=6669 ; 2014 \mathrm{n}=6100 ; 2017-18 \mathrm{n}=3319 \\
\text { d } 2011 \mathrm{n}=6677 ; 2014 \mathrm{n}=6104 ; 2017-18 \mathrm{n}=3321 \\
\text { e } 2011 \mathrm{n}=6672 ; 2014 \mathrm{n}=6092 ; 2017-18 \mathrm{n}=3321 . \\
\text { f } 2011 \mathrm{n}=6678 ; 2014 \mathrm{n}=6105 ; 2017-18 \mathrm{n}=3321\end{array}$} \\
\hline
\end{tabular}

The current study estimated the vaccination coverage among 12-59 months aged children in Bangladesh over three time periods from the data of BDHS (2011, 2014, and 2017-18). Though in 2014 the coverage of vaccination (85.1\%) is quite lower than the previous DHS 2011 (88.1\%), the status of full vaccination has increased significantly over time (89.2\% in 2017-18) $(p<0.001)$. The vaccination coverage which was reported either from vaccination cards or by mother recall has been also significantly increased over time. In 2011, the coverage of BCG was 97.2\%, in 2014 it was 96.8\% and while in 2017-18 the coverage 
increased to 98.4\%. All three doses of polio (Polio 1, Polio2, Polio 3) vaccines were observed to be increased over time and a significant increment $(\mathrm{p}<0.001)$ was seen in the full coverage of OPV (1-3) though a little decrease has been observed in 2014 DHS. The coverage of all doses of DPT (DPT 1, DPT 2, DPT 3), as well as the full coverage of DTP, were also increased from 2011 to $2017-18$ with a little decrease in 2014 ( $\mathrm{p}<0.001)$ (Table 2).

In Fig 1, probable cohort effects were depicted using the entire vaccination rate, which was plotted by the children's birth year (12-59 months old) using all available sources of data from 2006 to 2018. The figure shows that the trend of getting full vaccination by birth year quite fluctuates, and the coverage of the full vaccination increased about $1.6 \%$ over the thirteen years of birth cohort (88.9\% among children born in 2006 vs 90.5\% born in 2018).

Fig 2 depicts the geographical pattern of the change rate in full vaccination coverage throughout three survey periods. While most divisions saw a reduction in full vaccination coverage over time from 2011 to 2014, only Dhaka division (2.0 percent) saw a positive change rate, while Sylhet division had the worst scenario (-13.7\%). Interestingly, from 2014 to 2017-18, all divisions experienced an increase in vaccination status except Dhaka division $(-0.2 \%)$, while the highest improvement regarding the change rate was found in Sylhet division (19.5\%).

\section{Table 3. Bivariate distribution of basic vaccination coverage (full) by socio-demographic variables among children aged 12-59 months in Bangladesh using BDHS 2011, 2014 \& 2017-18 surveys}




\begin{tabular}{|c|c|c|c|c|c|c|c|c|c|}
\hline \multirow[t]{3}{*}{ Variables } & \multicolumn{9}{|c|}{ Fully vaccinated } \\
\hline & \multicolumn{3}{|c|}{2011} & \multicolumn{3}{|c|}{2014} & \multicolumn{3}{|c|}{ 2017-18 } \\
\hline & $\mathrm{n}$ & $\%(95 \% \mathrm{CI})$ & $\mathrm{p}$ & $\mathrm{n}$ & $\%(95 \% \mathrm{CI})$ & $\mathrm{p}$ & $\mathrm{n}$ & $\%(95 \% \mathrm{CI})$ & $\mathrm{p}$ \\
\hline \multicolumn{10}{|l|}{ Sex of child } \\
\hline Female & 2850 & $\begin{array}{c}86.8(85.6 \\
88.0)\end{array}$ & \multirow[t]{2}{*}{0.001} & 2530 & $\begin{array}{c}84.9(83.5 \\
86.1)\end{array}$ & \multirow[t]{2}{*}{0.658} & 1417 & $\begin{array}{c}89.5(87.9 \\
91.0)\end{array}$ & \multirow[t]{2}{*}{0.532} \\
\hline Male & 3036 & $\begin{array}{c}89.4(88.3 \\
90.4)\end{array}$ & & 2664 & $\begin{array}{c}85.3(84.0 \\
86.5)\end{array}$ & & 1544 & $\begin{array}{c}88.8(87.2 \\
90.3)\end{array}$ & \\
\hline \multicolumn{10}{|l|}{ Current age of child } \\
\hline $12-23$ & 1332 & $\begin{array}{c}86.2(84.3 \\
87.8)\end{array}$ & \multirow[t]{4}{*}{0.022} & 1285 & $\begin{array}{c}82.5(80.5 \\
84.4)\end{array}$ & \multirow[t]{4}{*}{0.007} & 1467 & $\begin{array}{c}88.1(86.4 \\
89.6)\end{array}$ & \multirow[t]{4}{*}{0.040} \\
\hline $23-35$ & 1369 & $\begin{array}{c}87.8(86.1 \\
89.4)\end{array}$ & & 1330 & $\begin{array}{c}86.5(84.6 \\
88.1)\end{array}$ & & 1494 & $\begin{array}{c}90.3(88.7 \\
91.3)\end{array}$ & \\
\hline $36-47$ & 1631 & $\begin{array}{c}88.9(87.3 \\
90.3)\end{array}$ & & 1285 & $\begin{array}{c}85.0(83.1 \\
86.8)\end{array}$ & & 0 & 0.0 & \\
\hline $48-59$ & 1554 & $\begin{array}{c}89.4(87.8 \\
90.8) \\
\end{array}$ & & 1294 & $\begin{array}{c}86.3(84.5 \\
88.0) \\
\end{array}$ & & 0 & 0.0 & \\
\hline \multicolumn{10}{|l|}{ Maternal age } \\
\hline$<24$ years & 2706 & $\begin{array}{c}88.7(87.5 \\
89.8) \\
\end{array}$ & \multirow[t]{3}{*}{0.001} & 2390 & $\begin{array}{c}85.2(83.8 \\
86.5) \\
\end{array}$ & \multirow[t]{3}{*}{0.051} & 1470 & $\begin{array}{c}88.0(86.3 \\
89.5) \\
\end{array}$ & \multirow[t]{3}{*}{0.980} \\
\hline 24-34 years & 2622 & $\begin{array}{c}88.6(87.3 \\
89.7)\end{array}$ & & 2361 & $\begin{array}{c}85.6(84.3 \\
86.9)\end{array}$ & & 1294 & $\begin{array}{c}90.4(88.8 \\
91.9)\end{array}$ & \\
\hline$>34$ years & 558 & $\begin{array}{c}83.7(80.6 \\
86.3)\end{array}$ & & 443 & $\begin{array}{c}81.6(78.0 \\
84.7)\end{array}$ & & 197 & $\begin{array}{c}89.5(84.5 \\
93.1)\end{array}$ & \\
\hline \multicolumn{10}{|l|}{ Maternal education } \\
\hline No education & 1021 & $\begin{array}{c}77.2(74.9 \\
79.4)\end{array}$ & \multirow[t]{4}{*}{$<0.001$} & 724 & $\begin{array}{c}74.1(71.2 \\
76.8)\end{array}$ & \multirow[t]{4}{*}{$<0.001$} & 170 & $\begin{array}{c}78.3(72.1 \\
83.5)\end{array}$ & \multirow[t]{4}{*}{$<0.001$} \\
\hline Primary & 1786 & $\begin{array}{c}86.5(85.0 \\
87.9)\end{array}$ & & 1381 & $\begin{array}{c}80.6(77.8 \\
81.6)\end{array}$ & & 779 & $\begin{array}{c}84.4(81.9 \\
86.6)\end{array}$ & \\
\hline Secondary & 2538 & $\begin{array}{c}92.8(91.7 \\
93.7)\end{array}$ & & 2506 & $\begin{array}{c}89.6(88.4 \\
90.7)\end{array}$ & & 1456 & $\begin{array}{c}91.2(90.3 \\
93.1)\end{array}$ & \\
\hline Higher & 541 & $\begin{array}{c}97.5(95.7 \\
98.6) \\
\end{array}$ & & 583 & $\begin{array}{c}94.3(92.1 \\
96.0) \\
\end{array}$ & & 556 & $\begin{array}{c}95.0(92.9 \\
96.6) \\
\end{array}$ & \\
\hline \multicolumn{10}{|l|}{ Wealth index } \\
\hline Poor & 2309 & $\begin{array}{c}83.2(81.8 \\
84.6)\end{array}$ & \multirow[t]{3}{*}{$<0.001$} & 1986 & $\begin{array}{c}79.8(78.1 \\
81.3)\end{array}$ & \multirow[t]{3}{*}{$<0.001$} & 1212 & $\begin{array}{c}86.9(85.0 \\
88.6)\end{array}$ & \multirow[t]{3}{*}{0.001} \\
\hline Middle & 1130 & $\begin{array}{c}89.6(87.8 \\
91.2)\end{array}$ & & 1028 & $\begin{array}{c}87.0(85.0 \\
88.9)\end{array}$ & & 522 & $\begin{array}{c}90.0(87.2 \\
92.3)\end{array}$ & \\
\hline Rich & 2447 & $\begin{array}{c}92.6(91.5 \\
93.6)\end{array}$ & & 2180 & $\begin{array}{c}89.6(88.3 \\
90.7)\end{array}$ & & 1227 & $\begin{array}{c}91.2(91.2 \\
90.4)\end{array}$ & \\
\hline \multicolumn{10}{|l|}{ Place of residence } \\
\hline Urban & 1862 & $\begin{array}{c}90.3(89.0 \\
91.6)\end{array}$ & \multirow[t]{2}{*}{$<0.001$} & 1664 & $\begin{array}{c}86.5(85.0 \\
88.0)\end{array}$ & \multirow[t]{2}{*}{0.030} & 1022 & $\begin{array}{c}89.3(87.3 \\
91.0)\end{array}$ & 0.953 \\
\hline Rural & 4024 & $\begin{array}{c}87.2(86.1 \\
88.1)\end{array}$ & & 3530 & $\begin{array}{c}84.4(83.3 \\
85.5)\end{array}$ & & 1939 & $\begin{array}{c}89.1(87.7 \\
90.4)\end{array}$ & \\
\hline Media access & & & & & & & & & \\
\hline $\begin{array}{l}\text { Never/less than } \\
\text { once }\end{array}$ & 2850 & $\begin{array}{c}84.7(83.4 \\
85.9)\end{array}$ & $<0.001$ & 2388 & $\begin{array}{c}79.9(78.4 \\
81.3)\end{array}$ & $<0.001$ & 1310 & $\begin{array}{c}87.3(85.5 \\
89.0)\end{array}$ & 0.002 \\
\hline At least once a week & 3036 & $\begin{array}{c}91.7(90.7 \\
92.6)\end{array}$ & & 2806 & $\begin{array}{c}90.0(88.9 \\
91.0)\end{array}$ & & 1651 & $\begin{array}{c}90.7(89.2, \\
92.0)\end{array}$ & \\
\hline Place of delivery & & & & & & & & & \\
\hline Home & 4278 & $86.2(85.2$ & $<0.001$ & 1568 & $81.7(79.8$ & $<0.001$ & 1452 & $86.3(84.6$ & $<0.001$ \\
\hline
\end{tabular}




\begin{tabular}{|c|c|c|c|c|c|c|c|c|c|}
\hline & & 87.1) & & & 83.4) & & & 87.9) & \\
\hline Health facility & 1596 & $\begin{array}{c}93.9(92.6 \\
95.0) \\
\end{array}$ & & 1043 & $\begin{array}{c}89.1(87.2 \\
90.8) \\
\end{array}$ & & 1502 & $\begin{array}{c}92.1(90.6 \\
93.3) \\
\end{array}$ & \\
\hline Others $^{\#}$ & 12 & $\begin{array}{c}92.3(62.1 \\
99.6)\end{array}$ & & 2583 & $\begin{array}{c}85.7(84.4 \\
86.9)\end{array}$ & & 7 & $\begin{array}{c}87.5(46.7 \\
99.3)\end{array}$ & \\
\hline \multicolumn{10}{|l|}{ ANC visit } \\
\hline Less than 3 & 3538 & $\begin{array}{c}86.9(85.8 \\
88.0)\end{array}$ & \multirow[t]{3}{*}{$<0.001$} & 1625 & $\begin{array}{c}81.9(80.2 \\
83.6)\end{array}$ & \multirow[t]{3}{*}{$<0.001$} & 1346 & $\begin{array}{c}86.1(84.2 \\
87.7)\end{array}$ & \multirow[t]{3}{*}{$<0.001$} \\
\hline At least 4 visits & 1317 & $\begin{array}{c}94.1(92.7 \\
95.2)\end{array}$ & & 844 & $\begin{array}{c}90.9(88.8 \\
92.6)\end{array}$ & & 1438 & $\begin{array}{c}92.6(91.2 \\
93.8)\end{array}$ & \\
\hline Others $\# \#$ & 1031 & $\begin{array}{c}85.3(83.2 \\
87.3)\end{array}$ & & 2725 & $\begin{array}{c}85.3(84.1 \\
86.5)\end{array}$ & & 177 & $\begin{array}{c}86.8(81.2 \\
90.9)\end{array}$ & \\
\hline \multicolumn{10}{|c|}{ Number of children } \\
\hline Single & 1762 & $\begin{array}{c}91.4(90.0 \\
92.6)\end{array}$ & \multirow[t]{3}{*}{$<0.001$} & 1774 & $\begin{array}{c}87.7(86.2 \\
89.1)\end{array}$ & \multirow[t]{3}{*}{$<0.001$} & 1151 & $\begin{array}{c}90.1(88.3 \\
91.7)\end{array}$ & \multirow[t]{3}{*}{0.126} \\
\hline Two children & 2064 & $\begin{array}{c}89.3(87.9 \\
90.5)\end{array}$ & & 1851 & $\begin{array}{c}87.5 \text { (86.0 } \\
88.9)\end{array}$ & & 1032 & $\begin{array}{c}89.4(87.5 \\
91.1) \\
\end{array}$ & \\
\hline More than two & 2060 & $\begin{array}{c}84.5 \text { (83.0 } \\
85.9)\end{array}$ & & 1569 & $\begin{array}{c}79.8(77.9 \\
81.5) \\
\end{array}$ & & 778 & $\begin{array}{c}87.4(85.0 \\
89.5)\end{array}$ & \\
\hline Total & 5886 & $\begin{array}{c}88.1(87.3 \\
88.9)\end{array}$ & & 5194 & $\begin{array}{c}85.1(84.2 \\
86.0)\end{array}$ & & 2961 & $\begin{array}{c}89.2(88.0 \\
90.2)\end{array}$ & \\
\hline
\end{tabular}

In the current study, as children's age rise, so does the prevalence of full vaccination coverage, and age is substantially associated with full vaccination coverage in all survey periods (all $\mathrm{p}<0.05$ ). Maternal age and maternal education had also a significant association with children's full vaccination coverage. The highest percentage of full vaccination coverage among children was observed when their mothers' age was between 24-34 years. Full vaccination coverage was also increased over the period with the increases of maternal educational level. In the present study, most of the children who got full vaccination were from urban areas $(90.3 \%, 86.5 \%$, and $89.3 \%$ respectively in three waves of BDHS). The coverage of full vaccination was also significantly associated with the media access, place of delivery, ANC visit (all p<0.05; Table 3).

Table 4. Regression analysis showing the associations between full vaccination and sociodemographic variables using BDHS 2011, 2014 \& 2017-18 surveys 


\begin{tabular}{|c|c|c|c|c|c|c|}
\hline \multirow[t]{2}{*}{ Variables } & \multicolumn{2}{|l|}{2011} & \multicolumn{2}{|c|}{2014} & \multicolumn{2}{|c|}{$2017-18$} \\
\hline & AOR $(95 \% \mathrm{CI})$ & p-value & AOR (95\% CI) & p-value & AOR (95\% CI) & p-value \\
\hline \multicolumn{7}{|l|}{ Sex of child } \\
\hline Male & Ref & & Ref & & Ref & \\
\hline Female & $0.9(0.8,1.0)$ & 0.078 & $1.0(0.9,1.2)$ & 0.846 & $1.2(0.9,1.5)$ & 0.149 \\
\hline \multicolumn{7}{|l|}{ Current age of child } \\
\hline $12-23$ & Ref & & Ref & & Ref & \\
\hline $23-35$ & $1.6(1.3,1.9)$ & $<0.001$ & $1.6(1.3,2.0)$ & $<0.001$ & $1.4(1.1,1.7)$ & 0.005 \\
\hline $36-47$ & $2.0(1.6,2.4)$ & $<0.001$ & $2.5(0.3,23.8)$ & 0.428 & - & - \\
\hline $48-59$ & $2.17(1.7,2.7)$ & $<0.001$ & $2.9(0.3,27.5)$ & 0.360 & - & - \\
\hline \multicolumn{7}{|l|}{ Maternal age } \\
\hline$<24$ years & Ref & & Ref & & Ref & \\
\hline 24-34 years & $1.1(0.9,1.4)$ & 0.314 & $1.3(1.1,1.6)$ & 0.003 & $1.5(1.1,2.0)$ & 0.008 \\
\hline$>34$ years & $0.9(0.7,1.3)$ & 0.703 & $1.5(1.1,2.0)$ & 0.015 & $1.8(1.1,3.2)$ & 0.032 \\
\hline \multicolumn{7}{|l|}{ Maternal education } \\
\hline No education & Ref & & Ref & & Ref & \\
\hline Primary & $2.0(1.7,2.4)$ & $<0.001$ & $1.5(1.3,1.8)$ & $<0.001$ & $2.1(1.5,2.8)$ & $<0.001$ \\
\hline Secondary & $3.4(2.8,4.3)$ & $<0.001$ & $2.6(2.1,3.1)$ & $<0.001$ & $3.6(2.6,4.9)$ & $<0.001$ \\
\hline Higher & $7.2(4.0,12.9)$ & $<0.001$ & $4.0(2.7,5.9)$ & $<0.001$ & $5.8(3.5,9.6)$ & $<0.001$ \\
\hline \multicolumn{7}{|l|}{ Wealth index } \\
\hline Poor & Ref & & Ref & & Ref & \\
\hline Middle & $1.3(1.1,1.7)$ & 0.009 & $1.2(0.9,1.5)$ & 0.054 & $1.2(0.8,1.6)$ & 0.380 \\
\hline Rich & $1.6(1.2,2.0)$ & $<0.001$ & $1.2(0.9,1.5)$ & 0.100 & $1.0(0.7,1.4)$ & 0.911 \\
\hline \multicolumn{7}{|l|}{ Place of residence } \\
\hline Urban & $1.5(1.3,1.8)$ & $<0.001$ & $1.4(1.2,1.7)$ & $<0.001$ & $1.4(1.1,1.7)$ & 0.006 \\
\hline Rural & Ref & & Ref & & Ref & \\
\hline \multicolumn{7}{|l|}{ Media access } \\
\hline Never/ less than once & Ref & & Ref & & Ref & \\
\hline At least once a week & $1.2(0.9,1.4)$ & 0.131 & $1.6(1.4,2.0)$ & $<0.001$ & $1.0(0.8,1.3)$ & 0.813 \\
\hline \multicolumn{7}{|l|}{ Place of delivery } \\
\hline Home & Ref & & Ref & & Ref & \\
\hline Health facility & $1.5(1.2,1.9)$ & 0.001 & $1.2(0.9,1.5)$ & 0.222 & $1.4(1.1,1.8)$ & 0.017 \\
\hline Others $^{\#}$ & $1.3(0.2,10.8)$ & 0.780 & $0.8(0.1,7.1)$ & 0.818 & $0.9(0.1,7.1)$ & 0.888 \\
\hline \multicolumn{7}{|l|}{ ANC visit } \\
\hline Less than 3 & Ref & & Ref & & Ref & \\
\hline At least 4 visits & $1.6(1.2,2.0)$ & 0.001 & $1.5(1.2,2.0)$ & 0.002 & $1.6(1.2,2.1)$ & $<0.001$ \\
\hline Others ${ }^{\# \#}$ & $0.8(0.6,0.9)$ & 0.011 & $0.8(0.6,1.3)$ & 0.377 & $1.0(0.6,1.6)$ & 0.930 \\
\hline \multicolumn{7}{|l|}{ Number of children } \\
\hline Single & Ref & & Ref & & Ref & \\
\hline Two & $1.2(0.9,1.4)$ & 0.180 & $1.1(0.9,1.3)$ & 0.564 & $1.1(0.8,1.4)$ & 0.590 \\
\hline More than two & $1.2(0.9,1.5)$ & 0.178 & $0.8(0.6,0.9)$ & 0.020 & $1.1(0.7,1.5)$ & 0.776 \\
\hline
\end{tabular}

The adjusted regression models of the present study demonstrate that age of the child, maternal age and education, wealth index, place of residence, media access, ANC visit of the 
mother and number of children were significantly associated with the coverage of the full vaccination in three different waves of BDHS. The full vaccination coverage was found to be increased significantly among the older child in all three BDHS. Maternal age was found to be significantly associated with the full vaccination coverage in two-time points except in 2011 . With the increase of the age of the mother, the vaccination coverage increased, the coverage of full vaccination was higher among the children with mothers aged $>34$ years, the likelihood of getting full vaccination were $1.5(\mathrm{AOR}=1.5,95 \% \mathrm{CI}: 1.1,2.0)$ and 1.8 (AOR=1.8, 95\% CI: 1.1, 3.2) times higher compared to children of mothers aged < 24 years in 2014 and 2017-18, respectively (Table 4).

The study found that mothers who have had primary, secondary, and higher education, their children experienced a better exposure to full vaccination than the mothers who have had no education in all three waves of BDHS. Wealth index also had an association with the coverage of full vaccination of the children. Children from rich families had 1.6 times higher odds of getting full vaccination in 2011 (AOR=1.6, 95\% CI: 1.2, 2.0), compared to those from families with poor wealth index, while wealth index was not found to be associated with vaccine status in 2014 and 2017-18. Children from urban areas were more likely to get fully vaccinated than their rural counterparts and the association is found to be significant in all three waves of BDHS (Table 4).

In the present study, place of delivery was also significantly associated with childhood full vaccination coverage in BDHS 2011 and 2017-18. The likelihood of getting full vaccination among children who got health facility during delivery was 1.5 and 1.4 times higher compared to those who were delivered at home in 2011 (AOR=1.5, 95\% CI: 1.2, 1.9) and 2017-18 $(\mathrm{AOR}=1.4,95 \% \mathrm{CI}: 1.1,1.8)$, respectively. We found that ANC visits at least 4 times significantly improved the full vaccination coverage like if the mother had at least 4 ANC visits then the likelihood of getting full vaccination was increased by 1.6 times in 2011 (AOR=1.6, 95\% CI: 1.2, 2.0), 1.5 times in 2014 (AOR=1.5, 95\% CI: 1.2, 2.0) and 1.6 times in 2017-18 (AOR=1.6, 95\% CI: 1.2, 2.1) (Table 4).

\section{Discussion}

The Sustainable Development Goal encourages countries and governments to take steps to ensure that their national immunization programs are fully vaccinated by 2030 [31]. Therefore, in order for Bangladesh to assess its progress toward full childhood immunization, it is critical to comprehend the 
factors that influence vaccination coverage over time. The goal of this study was to track the vaccination status of children aged 12 to 59 months and examine the factors that influence full immunization coverage. Overall, our findings show that the percentage of those who have had complete vaccination has increased significantly over time. This is substantiated by a recent Bangladeshi study that demonstrated a $20 \%$ increase in complete immunization coverage over time [20]. This increment in full vaccination coverage may possibly be attributable to conscious health policies such as the three nationwide supplementary immunization activities (SIAs) from 2000 to 2016 which were rolled out by the Bangladeshi government [21]. Despite the fact that full vaccination status for all vaccines increased significantly over time, BCG continuously had the greatest full vaccination coverage. This is consistent with the findings of Boulton et al., who found that BCG had the highest full vaccination coverage compared to the other vaccinations [20]. This conclusion could be explained by the fact that, unlike other vaccines that are given after a few weeks (such as OPV and DTP) or months (such as measles), BCG is given at birth, minimizing the risk of not getting immunized [32].

We also observed some divisional variations from our spatial analysis of the distribution of change rate in relation to childhood vaccination coverage. It can be observed from our study that, between 2011 to 2014, a positive change rate was observed in Dhaka division while the worst situation was found in Sylhet division. This is in agreement with the findings of Sheikh et al. [18]. This higher likelihood of incomplete vaccination in the Sylhet division was linked to the remote hilly and riverine nature of the area coupled with the fragile communication system of this area [18]. However, between 2014 to 2017-18, all divisions experienced an increase in vaccination status except Dhaka division, with the highest improvement in change rate being recorded in the Sylhet division.

Our findings also reveal that the factors associated with childhood vaccination coverage over time spread across child, maternal and contextual factors. Concerning the child factors, our analyses revealed that the current age of the child was significantly associated with full vaccination coverage. Thus, the likelihood of full vaccination increased with increased age in children. Hence, older children were more likely to be fully vaccinated. The findings align with evidence from DR Congo [3]. First, childhood vaccinations are scheduled and for that matter, it is expected that older children will be at a higher likelihood of being fully vaccinated. Moreover, Bangladesh has had a fair share of mass vaccination programs over the years [33]. Such mass vaccinations, in the perspective of Alfonso et al. [3], lead to catch-up vaccination with age, thereby predisposing older children to a higher possibility of being fully vaccinated.

In relation to maternal factors and childhood vaccination coverage, our findings revealed that maternal age and education were significantly associated with vaccination coverage over time. This result is substantiated by earlier studies conducted in Bangladesh [34,35], DR Congo [36], and Ethiopia [6]. Our findings revealed that children born to mothers older than 34 years were 1.8 times more likely to be fully vaccinated as compared to those born to mothers younger than age 24. Often, mothers younger than age 24 are inexperienced and therefore may not be knowledgeable about the timing and relevance of ensuring that their children receive the full dose of vaccinations. Hence, explaining the lower odds of full vaccination coverage among children born to mothers younger than age 24 . Children born to mothers 
who had formal education had higher likelihoods of full vaccination coverage. A plausible justification of the effect of maternal education on childhood vaccination coverage could be that mothers with formal education are more likely to be knowledgeable about childhood vaccination, its benefits, as well as being aware of the schedules [37].

At the contextual level, urban residence, delivering at a place having health facilities, and attending at least 4 ANC visits were significantly associated with increased full vaccination coverage. This finding is consistent with studies from Bangladesh [3], as well as studies from Ethiopia [37] and Ghana [2].

Delivering at a health facility and attending at least 4 ANC visits provides mothers with the opportunity to be exposed to health education and sensitization messages about the importance of ensuring full vaccination of their children, as well as providing parents with vaccination schedules [37]. Another possible explanation for the findings is that mothers who receive more ANC visits may gain satisfaction with healthcare access which could potentially translate into higher vaccination coverage [38]. Concerning the urbanicity of our findings, it could be argued from the perspective that urban areas have a substantial proportion of health professionals and health facilities; therefore, more children in the urban areas are likely to have access to full vaccination coverage compared to their counterparts in the rural areas $[16,39]$.

\section{Strengths and limitations}

Our study has several strengths. The dataset employed in this study was nationally representative, ensuring that the findings may be applied to children throughout Bangladesh. In addition, the DHS dataset is a validated, repeatable, standardized, and very thorough survey. This, combined with the rigorous statistical procedures used, ensures the validity of our findings and the study's replicability in other scenarios. Nonetheless, the study has several intrinsic limitations, which should be taken into account when interpreting our findings. Causation cannot be established because of the BDHS's use of cross-sectional design. Furthermore, information about the status of child vaccination is dependent on either immunization cards or women's self-reports; hence, there is the possibility of recall bias, which could lead to an under-or overestimation of vaccination coverage.

\section{Conclusion}

According to the study, children's immunization rates have risen over time. The national trend of childhood immunization rates showed an increasing pattern as a result of various known child, maternal, and demographic factors. To increase childhood immunization in Bangladesh, we believe that EPIs, SIAs, and other full-childhood vaccine interventions should focus on children aged 12-23 months, those born to mothers with no formal education, and younger mothers (less than 24 years). In addition, if Bangladesh is to attain $100 \%$ immunization coverage, the government must step up efforts to increase facility births and ANC attendance. 


\section{Declarations}

Acknowledgement

The authors are grateful to the authority of BDHS.

Author contributions

Satyajit Kundu: Conceptualization, methodology, data curation, formal analysis, writing - original draft; Subarna Kundu: Conceptualization, methodology, data curation, formal analysis, writing - original draft; Bright Opoku Ahinkorah: Writing - original draft, review and editing; Abdul-Aziz Seidu: Writing original draft, review and editing; Joshua Okyere: Writing - original draft, review and editing; Susmita Ghosh: Writing - original draft, review and editing; Ahmed Hossain: Writing - original draft, review and editing; Najim Z. Alshahrani: Writing - original draft, review and editing; Md. Hasan Al Banna: Writing original draft, review and editing. All authors read and approved the final manuscript.

Competing interests

The authors have declared that no competing interests exist.

Funding

The author(s) received no specific funding for this work.

\section{References}

1. Amadu I, Seidu A-A, Duku E, Okyere J, Hagan JE, Hormenu T, et al. The Joint Effect of Maternal Marital Status and Type of Household Cooking Fuel on Child Nutritional Status in Sub-Saharan Africa: Analysis of Cross-Sectional Surveys on Children from 31 Countries. Nutrients. 2021;13: 1541.

2. Budu E, Ahinkorah BO, Aboagye RG, Armah-Ansah EK, Seidu A-A, Adu C, et al. Maternal healthcare utilsation and complete childhood vaccination in sub-Saharan Africa: a cross-sectional study of 29 nationally representative surveys. BMJ Open. 2021;11: e045992.

3. Alfonso VH, Bratcher A, Ashbaugh H, Doshi R, Gadoth A, Hoff N, et al. Changes in childhood vaccination coverage over time in the Democratic Republic of the Congo. PLoS One. 2019;14: e0217426.

4. Kim YE. Determinants of childhood vaccination in Nagaland, India: a cross-sectional study with multilevel modelling. BMJ Open. 2021;11: e045070.

5. WHO. Global and regional immunization profile. 2020. Available:

https://www.who.int/immunization/monitoring_surveillance/data/gs_gloprofile.pdf 
6. Fenta SM, Fenta HM. Individual and community-level determinants of childhood vaccination in Ethiopia. Arch Public Heal. 2021;79: 1-11.

7. Zenbaba D, Sahiledengle B, Debela MB, Tufa T, Teferu Z, Lette A, et al. Determinants of Incomplete Vaccination Among Children Aged 12 to 23 Months in Gindhir District, Southeastern Ethiopia: Unmatched Case-Control Study. Risk Manag Healthc Policy. 2021;14: 1669.

8. Casula M, Toth F. The 2017 Italian reform on mandatory childhood vaccinations: Analysis of the policy process and early implementation. Health Policy (New York). 2021;125: 7-11.

9. Li X, Mukandavire C, Cucunubá ZM, Londono SE, Abbas K, Clapham HE, et al. Estimating the health impact of vaccination against ten pathogens in 98 low-income and middle-income countries from 2000 to 2030: a modelling study. Lancet. 2021;397: 398-408.

10. Nour TY, Farah AM, Ali OM, Osman MO, Aden MA, Abate KH. Predictors of immunization coverage among 12-23 month old children in Ethiopia: systematic review and meta-analysis. BMC Public Health. 2020;20: 1-19.

11. Mekonnen ZA, Gelaye KA, Were MC, Tilahun B. Timely completion of vaccination and its determinants among children in northwest, Ethiopia: a multilevel analysis. BMC Public Health. 2020;20: $1-13$.

12. WHO. Improving influenza vaccine virus selection: Report of the 4th WHO informal consultation, Hong Kong SAR, China, 18-20 November 2015. World Health Organization; 2015.

13. VanderEnde K, Gacic-Dobo M, Diallo MS, Conklin LM, Wallace AS. Global routine vaccination coverage-2017. Morb Mortal Wkly Rep. 2018;67: 1261.

14. WHO. Early childhood development and disability: A discussion paper. 2012.

15. Jalloh MF, Bennett SD, Alam D, Kouta P, Lourenço D, Alamgir M, et al. Rapid behavioral assessment of barriers and opportunities to improve vaccination coverage among displaced Rohingyas in Bangladesh, January 2018. Vaccine. 2019;37: 833-838.

16. Budu E, Darteh EKM, Ahinkorah BO, Seidu A-A, Dickson KS. Trend and determinants of complete vaccination coverage among children aged 12-23 months in Ghana: Analysis of data from the 1998 to 2014 Ghana Demographic and Health Surveys. PLoS One. 2020;15: e0239754.

17. Ushie BA, Fayehun OA, Ugal DB. Trends and patterns of under-5 vaccination in N igeria, 19902008: what manner of progress? Child Care Health Dev. 2014;40: 267-274.

18. Sheikh N, Sultana M, Ali N, Akram R, Mahumud RA, Asaduzzaman M, et al. Coverage, timelines, and determinants of incomplete immunization in Bangladesh. Trop Med Infect Dis. 2018;3: 72. 
19. NIPORT, Mitra and Associates and II. Bangladesh Demographic and Health Survey 2014. Dhaka, Bangladesh: NIPORT, Mitra and Associates, and ICF International. 2016. Available: http://dhsprogram.com/pubs/pdf/FR311/FR311.pdf.

20. Boulton ML, Carlson BF, Power LE, Wagner AL. Socioeconomic factors associated with full childhood vaccination in Bangladesh, 2014. Int J Infect Dis. 2018;69: 35-40.

21. Khanal S, Bohara R, Chacko S, Sharifuzzaman M, Shamsuzzaman M, Goodson JL, et al. Progress toward measles elimination-Bangladesh, 2000-2016. MMWR Morb Mortal Wkly Rep. 2017;66: 753.

22. Unicef. Committing to child survival: a promise renewed. eSocialSciences; 2015.

23. Cutts FT, Claquin P, Danovaro-Holliday MC, Rhoda DA. Monitoring vaccination coverage: defining the role of surveys. Vaccine. 2016;34: 4103-4109.

24. NIPORT, Ministry of Health and Family Welfare and I. Bangladesh Demographic and Health Survey 2017-18. Dhaka, Bangladesh: NIPORT/ICF. 2020. Available:

https://www.dhsprogram.com/pubs/pdf/FR344/FR344.pdf.

25. NIPORT, Mitra and Associates and II. Bangladesh Demographic and Health Survey 2011. Dhaka, Bangladesh: NIPORT, Mitra and Associates, and ICF International. 2013. Available: http://dhsprogram.com/pubs/pdf/FR265/FR265.pdf.

26. Odusanya OO, Alufohai EF, Meurice FP, Ahonkhai VI. Determinants of vaccination coverage in rural Nigeria. BMC Public Health. 2008;8: 1-8.

27. Moran EB, Wagner AL, Asiedu-Bekoe F, Abdul-Karim A, Schroeder LF, Boulton ML. Socioeconomic characteristics associated with the introduction of new vaccines and full childhood vaccination in Ghana, 2014. Vaccine. 2020;38: 2937-2942.

28. Khan J, Shil A, Mohanty SK. Hepatitis B vaccination coverage across India: exploring the spatial heterogeneity and contextual determinants. BMC Public Health. 2019;19: 1-14.

29. Obanewa OA, Newell ML. The role of place of residency in childhood immunisation coverage in Nigeria: Analysis of data from three DHS rounds 2003-2013. BMC Public Health. 2020;20: 123.

30. Wikipedia. Mymensingh Division. 2021 [cited 15 Jun 2021]. Available: https://en.wikipedia.org/wiki/Mymensingh_Division

31. Ratzan SC, Bloom BR, El-Mohandes A, Fielding J, Gostin LO, Hodge JG, et al. The Salzburg statement on vaccination acceptance. J Health Commun. 2019;24: 581-583.

32. Herrera OR, Thornton TA, Helms RA, Foster SL. MMR Vaccine: When Is the Right Time for the Second Dose? J Pediatr Pharmacol Ther. 2015;20: 144-148. 
33. Nurunnabi ASM, Mozaffor M, Hossain MA, Sony SA. Mass Vaccination Programme: Public Health Success and Ethical Issues-Bangladesh Perspective. Bangladesh J Bioeth. 2018;9: 11-15.

34. Haque SMR, Bari W. Positive role of maternal education on measles vaccination coverage in Bangladesh. Int J Psychol Behav Sci. 2013;3: 11-17.

35. Rahman M, Obaida-Nasrin S. Factors affecting acceptance of complete immunization coverage of children under five years in rural Bangladesh. Salud Publica Mex. 2010;52: 134-140.

36. Acharya P, Kismul H, Mapatano MA, Hatløy A. Individual-and community-level determinants of child immunization in the Democratic Republic of Congo: a multilevel analysis. PLoS One. 2018;13: e0202742.

37. Kinfe $\mathrm{Y}, \mathrm{Gebre} \mathrm{H}$, Bekele A. Factors associated with full immunization of children 12-23 months of age in Ethiopia: A multilevel analysis using 2016 Ethiopia Demographic and Health Survey. PLoS One. 2019;14: e0225639.

38. Ntenda PAM. Factors associated with non-and under-vaccination among children aged 12-23 months in Malawi. A multinomial analysis of the population-based sample. Pediatr Neonatol. 2019;60: 623-633.

39. Tsawe M, Susuman AS. Determinants of access to and use of maternal health care services in the Eastern Cape, South Africa: a quantitative and qualitative investigation. BMC Res Notes. 2014;7: 1-10.

\section{Figures}




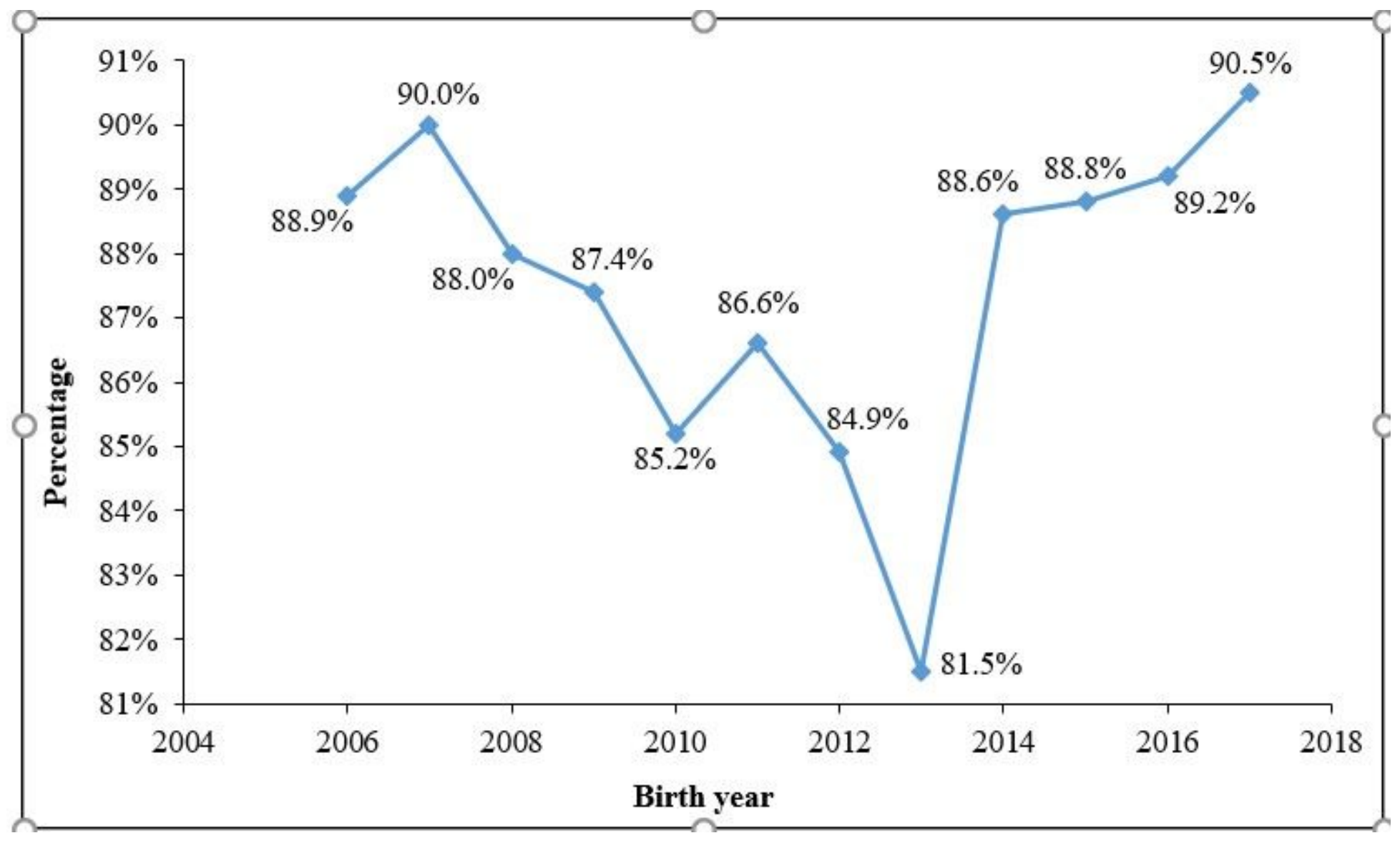

Figure 1

Percentage of fully vaccinated children (12-59 months old) in Bangladesh by birth cohort from 2006 to 2017. 


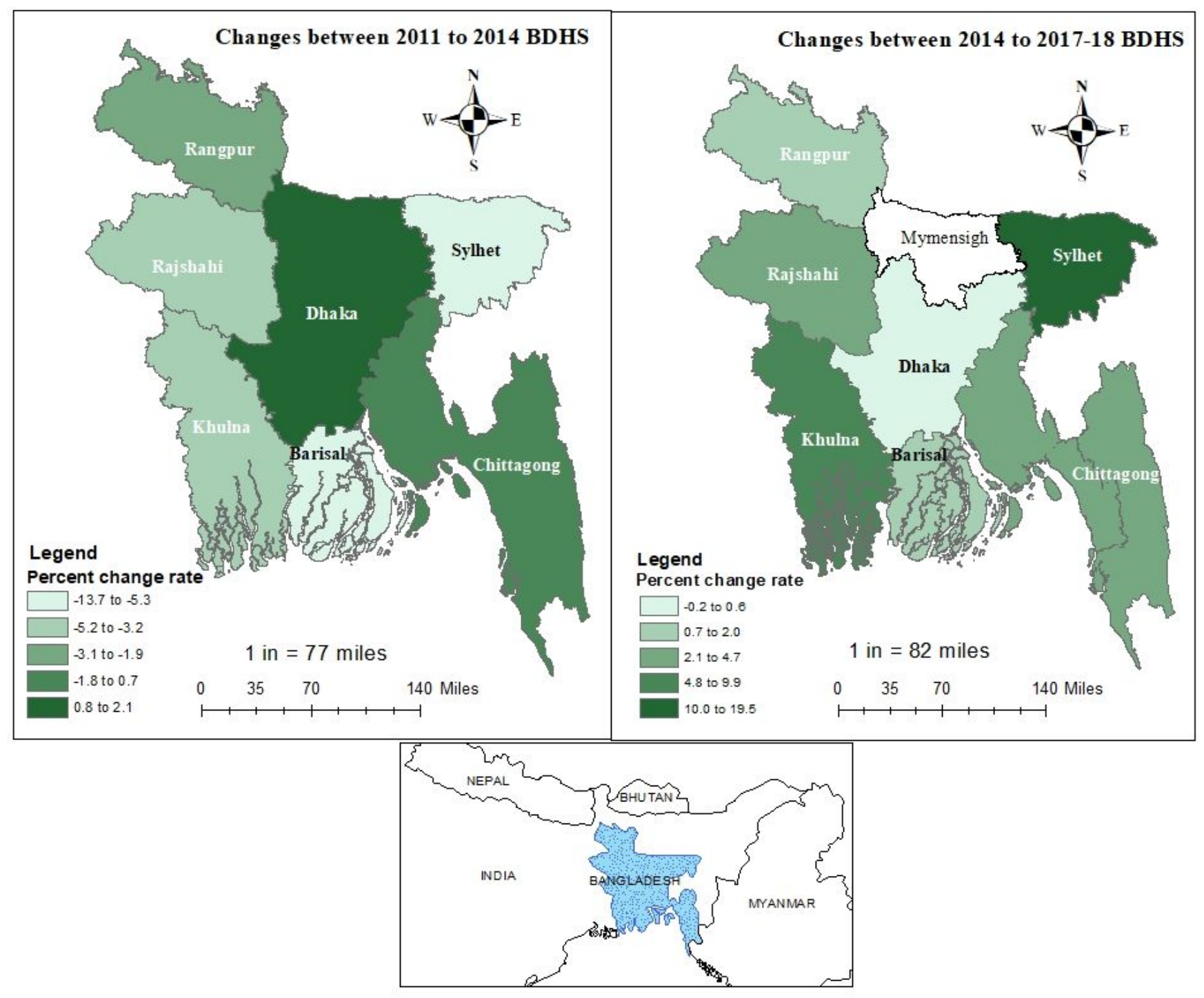

Figure 2

Spatial distribution of change rate in vaccination status among children aged 12-59 months old from BDHS 2011 to 2014 and BDHS 2014 to 2017-18. Mymensingh division was separated from Dhaka division in 2015 composing of four districts of northern part of Bangladesh. Hence, the change rate of vaccination status for Mymensingh division is not shown in the map. 